REVISTA MATEMATICA de la

Universidad Complutense de Madrid

Volumen 2, número suplementario, 1989

http://dx.doi.org/10.5209/rev REMA.1989.v2.18078

\title{
Structure of measures on topological spaces
}

\author{
JOSE L. de MARIA and BALTASAR RodRIgUez-SALINAS
}

\begin{abstract}
The Radon spaces of type (i), i.e., topological spaces for which every finite Borel measure on $\Omega$ is $\tau$-additive and -regular are characterized. The class of these spaces is very wide and in particular it contains the Radon spaces. We extend the results of Marczewski and Sikorski to the $\sigma$-metrizable spaces and to the subsets of the Banach spaces endowed with the weak topology. Finally, the completely additive families of measurable subsets related with the works of Hansell, Koumoullis and Fremlin are studied.
\end{abstract}

\section{INTRODUCTION}

The modern Measure Theory starts with the construction of the Borel measures on the $\sigma$-algebra of the Borel sets of $\mathbb{R}$. Two important facts are to be noted: 1) The measures are defined on a $\sigma$-algebra. 2) They are countably additive.

The study of the measures on $\mathbb{R}$ is the origin for later study of measures on metric spaces started by Caratheodory and also the study of measures on locally compact spaces with the brilliant construction of the Haar measure.

Some authors, Bourbaki among others, thought the frame of locally compact spaces wide enought for a satisfactory measure theory. But the 60-70's represent such a radical change in the way of thinking about measure theory in topological spaces, that even Bourbaki publishes in 1969 a volume about measures defined on non locally compact spaces. This different point of view is due to the new relationship between mathematical analysis and probability calculus, in which measure theory had an important development some years before, by virtue of the papers of P.J. Daniel, H. Steinhaus, B. Jessen, P. Lévy, N. Wiener, Ju. V. Prokhorov, L. de Cam, R. A. Minlos, etc. This above mentioned book of Bourbaki generated the general measure theory on topological spaces.

Supported in part by CAICYT 0338-84

1980 Mathematics Subject Classification (1985 revision): $28 \mathrm{C} 15,46 \mathrm{G} 12$.

Editorial de la Universidad Complutense. Madrid 1989. 
One of the most distinguished measure theory is due to L. Schwartz, who gave a lecture in 1964, in the Gulbenkian Institute of Lisbon, about the Radon measure theory on non-locally compact spaces. In 1965 he gave another lecture in the Tata Institute in Bombay where he developed the theory. Finally in 1973 appears the desired book "Radon Measures on Arbitrary Topological Spaces and Cylindrical Measures" which contain almost all of Bourbaki's Chapter IX on integration. At the same time, in 1964, we gave a communication about the measure theory on topological spaces at the $\mathrm{V}$ Reunion de Matemáticos Españoles. Rodriguez-Salinas gave the main lecture in the Primeras Jornadas Luso-Españolas in 1972 precisely on the same subject and also in the Gulbenkian Institute. Afterwards he continued working and publishing papers on the same subject.

The relationship between topology and measure theory is described in term of the regularity properties and in particular the "outher" and "inner" regularity. A measure $\mu$ defined on the $\sigma$-algebra of the Borel sets of a topological space is said to be outer regular if the measure of each Borel set is the infimum of the measures of all open sets containing it. A measure $\mu$ in the same conditions is said to be inner regular if the measure of each Borel set is the supremum of the measures of all the compact sets contained in it.

Among the first contributions on the regularity of measures, we must point out the papers of A. D. Alexandroff (1940-1950), P. R. Halmos and I. von Neumann (1950), E. Marczewski (1953), C. Ryll Nardzewski (1953), B. V. Gnedenko and A. N. Kolmogorov (1954), and D. Blackwell (1955). Alexandroff emphasizes inner regularity and proves that the measures on a Polish space are inner regular. This result was later found again by Prokhorov in 1956.

Radon measures on topological spaces can be defined in different ways. The Schwartz's method is one of them. In particular Radon measures on completely regular spaces have been studied, apart from Schwartz, by A.D. Alexandroff, V. S. Varadarajan and K. Zizi. P. A. Meyer has defined Radon measures on Hausdorff spaces by use of the concept of compactology of $A$. Weil.

Radon measure theory is based on inner regularity, i.e. by use of the inner approximation of the measure by means of the measures of the compact sets. The first problem that it appears is that it is only possible to induce measures in measurable subsets. Hence it is necessary to substitute this inner regularity by an inner regularity with respect to a class $(, \mathscr{C})$ ) of closed sets. This class could be the class $(\mathscr{F})$ of all closed sets or some particular class such as the class $\left(\mathscr{X} \mathscr{K}_{m}\right)$ of all the metrizable compact sets. In this plurality rests the usefulness of the Radon mesaures of type ( $\mathscr{O})$. But the compactenes must be substituted by something which plays a similar role, this is the concept of $\mu$-compactness introduced by us in 1964. The Radon measures of type ( $\%$ ) allow to describe the 
structure of measures on the most important topological spaces. In this lecture we are going to discuss this cuestion.

\section{BASIC CONCEPT}

Let $\tau$ be a real function, defined on the class $\mathscr{f}(\Omega)$ of a topological space $\Omega$. monotone and such that $\tau(\boldsymbol{\sigma})=0$. A subset $A$ of $\Omega$ is said to be $\tau$-compact if, for every open cover $G_{0}$ of $A$ and for every $\varepsilon>0$, there exists $\left\{G_{1}, \ldots, G_{n}\right\} \subset C_{0}$ such that

$$
\tau\left(A \backslash \bigcup_{1}^{n} G_{k}\right)<\varepsilon
$$

Obviously every compact set is $\tau$-compact and if $\tau(\emptyset)=0$ and $\tau(A)=1$ for every $A \neq \emptyset$, we have that each $\tau$-compact is compact.

Analogously the notion of $\mu$-compact set is also introduced when $\mu$ is measure defined on the $\sigma$-algebra of Borel sets of a topological space.

Let $\%$ be a class of closed sets in a topological space $\Omega$. Then a Borel measure $\mu$ on $A$ ( $\sigma$-algebra of Borel in $\Omega$ ) is said to be a Radon measure of type $(\not y)$ if

1) Every $H \in . \not$ is $\mu$-compact and $\mu(H)$ is finite.

2) $\mu(B)=\sup \{\mu(H): B \supset H \in \not \nVdash\}$, for every $B \in A$ ( $A$ can be substituted by a class $A D A$ )

In particular, if $\Omega$ is a Hausdorff space and $\mathscr{K}$ is the class of compacts of $\Omega$, the Radon measures of type $(K)$ are the usual Radon measures. (Sometimes it is required that the Radon measures are locally finite in order to assure that the measure of the compact sets is finite). Other important classes, as we have remarked already, are $/ /=\mathscr{K}_{\mathrm{m}}$ the class of the metrizable compact subsets and $y t=y$ the class of all closed sets. Every finite Radon measure of type $(\%)$ is a Radon measure of type $(-\not)$.

If $\mu^{*}$ is the outer measure associated to a locally finite Radon measure of type $(Y /)$, then a set $A$ is $\mu^{*}$-compact if and only if $\mu^{*}(A)$ is finite.

From now on we assume that all measures we consider are finite and that $\Omega$ is a topological space.

A Banach measure on $\Omega$ is a measure $\mu \neq 0$ on $\mathscr{f}(\Omega)$ such that $\mu(\omega)=0$ for every $\omega \in \Omega$. 
An Ulam measure on $\Omega$ is a Banach measure on $\Omega$ with values in $\{0,1\}$.

A cardinal $\alpha$ is real-measurable if there exists a Banach measure on some space $\Omega$ of cardinality $\alpha$.

A cardinal $\alpha$ is 2 -measurable if there exists an Ulam measure on some space $\Omega$ of cardinality $\alpha$.

The non 2-measurable cardinals are called non-measurable and the non real-measurable cardinals are called cardinals of measure zero.

The cardinal $c=2^{\kappa_{0}}$ is non-mesurable and with the Continuum Hypothesis, it is of measure zero. Moreover there exist axioms of Set Theory consistent with ZFC which assure that every cardinal is of measure zero. Such an example is given by the Godel Constructibily Axiom. Ulam proved in 1930 that every real-measurable cardinal is either $\leq 2^{\kappa}$ or else measurable (but not both).

In 1984 Marczewski and Sikorski [23] have proved that the existence of a dense set with cardinality of measure zero in a metric space is equivalent to the existence, for every finite Borel measure $\mu$ on $\Omega$, of a separable closed subset $F$ such that $\mu(\Omega \backslash F)=0$. They also proved that this last property is equivalent to the fact that every Borel measure on $\Omega$ has a proper support. These results can be completed in this way: Every Borel measure on a metric space $\Omega$ is a Radon measure of type (形) if and only if the weight of $\Omega$ is a cardinal of measure zero. The weight of a topological space $\Omega$ is the smallest cardinal of the dense subsets of $\Omega$.

For every Borel measure $\mu$ on a metric space $\Omega$, one has

$$
\mu(B)=\sup \{\mu(F): B \supset F \in \bar{f}\}
$$

for every Borel set B. So it is obvious that a Borel measure $\mu$ is Radon of type $(-\not)$ if and only if $\Omega$ is $\mu$-compact.

A topological space $\Omega$ is called a Radon space of type ( $\not t)$ if every Borel measure on $\Omega$ is a Radon measure of type ( $y)$. In particular, Radon spaces of type ( $\mathscr{K}$ ) coincide with the Radon spaces.

A subset $A$ of $\Omega$ is said universally Borel measurable (resp. universally Radon measurable of type $(y)$ ) if, for every Borel measure (resp. Radon measure of type $(\mathscr{H})) \mu$ on $\Omega$, there exist two Borel subsets $B, B^{\prime}$ such that $B \subset A \subset B^{\prime}$ and $\mu(B \backslash B)=0$

$\Omega$ is said universally measurable if every Radon measure of type $(j)$ on $\Omega$ 
is a Radon measure. This definition coincides with the usual one when $\Omega$ is a completely regular space. (See [36] 1.2.11,[30] Lemma 8). Complete metric spaces are universally measurable ([29] Corollary 5). So they are Radon spaces if and only if their weight is of measure zero. Hence we have a generalization of the similar property of Polish spaces.

A $\sigma$-algebra $-t$ of subsets of $\Omega$ is self-generative if a set $A$ belongs to $-t$ if and only if for every $x \in A$, there exists aneighborhood $V(x)$ such that $A \cap V(x)$ $E$. . The smallest self-generative $\sigma$-algebra $\delta$ which contains open sets is called the Spanish $\sigma$-algebra, the elements of $\delta$ are called the Spanish sets. In particular, if $\Omega$ is strongly Lindelöf then $\delta=\mu$

\section{RADON SPACES OF TYPE $(\mathscr{X})$}

Now we are going to study the structure of the Radon spaces of type ( $f$ ) in relation with the self-generative character of its $\sigma$-algebra of the measurable sets, and with a property, inspired from a Lemma of D. Montgomery [25]. We have called Flock spaces, the spaces which own this property. We also use the concept of $\mathrm{L}$-weight which is related with the weight of a topological space and the property of Lindelof.

1. Proposition.If $(G)_{\mathrm{a}}$ is a well-ordered family of open sets in $\Omega$, and $H_{\mathrm{a}}=G_{\mathrm{a}} \bigcup_{\beta<\mathrm{a}} G_{\mathrm{\beta}}$ and $E_{\mathrm{a}} \subset H_{\mathrm{a}}$ is a Spanish set, then the union $E=\bigcup_{\mathrm{a} \in \mathrm{A}} E_{\mathrm{a}}$ of each subfamily of $\left(E_{\mathrm{a}}\right)_{\mathrm{a}}$ is a Spanish set.

Proof. Let $G_{\mathrm{a}}^{\prime}=\cup G_{\beta<a}$. By transfinite induction we will prove that $E \cap G_{\mathrm{a}}^{\prime}$ is a Spanish set. Indeed, 1) $E \cap G_{1}^{\prime}=$ o. 2) If $E \cap G_{\mathrm{a}}^{\prime} \in \delta$ then

$$
E \cap G_{\mathrm{a}+1}^{\prime}=\left(E \cap G_{\mathrm{a}}^{\prime}\right) \cup E_{\mathrm{a}}^{\prime} \in \mathcal{\delta}
$$

with $E_{\mathrm{a}}^{\prime}=E_{\mathrm{a}}$ or $E_{\mathrm{a}}^{\prime}=$ o. 3) If $\alpha$ is a limit ordinal and we suppose that $E \cap G_{\beta}^{\prime} \in \mathrm{c}$ for every $\beta<\alpha$ then every $x \in E \cap G_{\alpha}^{\prime}$ has a neighborhood $V(x)=G_{\beta}^{\prime}$ with $\beta<\alpha$ such that

$$
\left(E \cap G_{\alpha}^{\prime}\right) \cap V(x)=E \cap G_{\beta}^{\prime} \in \mathcal{E}
$$

so, $E \cap G_{\mathrm{a}}^{\prime} \in \mathscr{S}$ and $E=E \cap \underset{\mathrm{a}}{\cup} G_{\mathrm{a}} \in \mathcal{S}$.

2. Corollary. Under the hypothesis of Proposition 1, every union $\underset{a \in A}{\cup} H_{\mathrm{a}}$ of a subfamily of $\left(H_{\alpha}\right)_{a}$ is a Spanish set.

3. Definition. $\Omega$ is called a Flock space if, for every well-ordered family $\left(G_{\alpha}\right)_{a}$ of open subsets of $\Omega$ and setting $H_{\mathrm{a}}=G_{\mathrm{a}} \backslash \backslash_{\beta<\mathrm{a}} G_{\beta}$, the union $\underset{\mathrm{a} \in A}{\cup} H_{\mathrm{a}}$ of any subfamily of $\left(H_{0}\right)_{\alpha}$ is a universally Borel measurable set. 
If $\Omega$ a metrizable space, from Lemma 2 [25], we get that every union $\cup H_{a}$ is a $F_{\mathrm{v}}$ set, so $\Omega$ is a Flock space. Also, every strongly Lindelōf space is a Flock space.

From Theorem 2.12.6 [28] and Corollary 47 [20] it follows that the measurable sets with respect to a Radon measure of type (f) constitute a self-generative $\sigma$-algebra which contains $2 \delta$. so every element of $\delta$ is universally Borel measurable of type $(T)$.

Let us prove that the $\sigma$-algebra of the measurable sets for a Radon measure $\mu$ of type $(A)$ is self-generative. For every $x \in A$ there exists a neighborhood $V(x)$ such that $A \cap V(x)$ is measurable, as $G=\cup V_{x \in A}(x)$ is $\mu$-compact there exist a sequence $\left(x_{n}\right) \subset A$ such that

$$
\mu\left(G \backslash \cup_{1}^{\infty}\left(x_{n}\right)\right)=0
$$

so $A \backslash \bigcup_{1}^{\infty}\left(x_{n}\right)$ is measurable, hence

$$
A=\bigcup_{1}^{\infty}\left(A \cap V^{\infty}\left(x_{n}\right)\right) \cup\left(A \backslash \cup_{1}^{\infty} V^{\circ}\left(x_{n}\right)\right)
$$

is also measurable since $A \cap V^{\circ}\left(x_{n}\right)=\left(A \cap V\left(x_{n}\right)\right) \cap V^{\circ}\left(x_{n}\right)$.

4. Definition. $\Omega$ has the $\alpha$-property of Lindelof, where $\alpha$ is a transfinite cardinal, if for every family $\left(G_{i}\right)_{i \in l}$ of open subsets of $\Omega$, there exists $J \subset I$, such that $\operatorname{card}(J) \leqslant \alpha$, and

$$
\cup G_{i \in I}=\cup_{i \in J} G_{i}
$$

If a base of the topology of $\Omega$ has cardinal $\alpha$, then $\Omega$ has the $\alpha$-property of Lindelöf.

The smallest cardinal $\alpha$ such that $\Omega$ has the $\alpha$-property of Lindelöf is called the $\mathrm{L}$-weight of $\Omega$.

5. Theorem. Let $\Omega$ be a Flock space whose L-weight is of measure zero and $\left(G_{\mathrm{a}}\right)_{\mathrm{a}}$ be a family of open sets in $\Omega$, then

$$
\mu\left(\cup G_{a}\right)=\sup \underset{J}{\sim}\left(\underset{a \in J}{\cup} G_{a}\right),
$$

for every Borel measure $\mu$ where the supremum is taken over all finite subsets $J$ of $A$.

Proof. By Zerme o theorem and as the L-weight of $\Omega$ is of measure zero, we can suppose that $A$ is well-ordered and its cardinal of measure zero. Let 


$$
H_{\mathrm{a}}=G_{\mathrm{a}} \backslash \cup G_{\mathrm{p}<\mathrm{a}}
$$

Since $\Omega$ is a Flock space, every union $\underset{\mathrm{a} \in A}{\cup} H_{\mathrm{a}}$ is an universally Borel measurable. Then the set function

$$
U(S)=\mu^{*}\left(\underset{a \in S}{\cup} H_{a}\right)
$$

is a finite measure defined over all subsets $S \subset A$, where $\mu^{*}$ designates the outer measure associated to $\mu$. As the cardinality of $A$ is of measure zero, there exists a countable set $S \subset A$ such that $\mathrm{v}(A)=\mathrm{v}(S)$, i.e., $\mu^{*}\left(\underset{a \in A}{\cup} H_{a}\right)=\mu^{*}\left(\cup_{a \in S} H_{a}\right)$.

Now,

$$
\mu\left(\cup_{a \in A} G_{a}\right)=\mu\left(\cup_{a \in A} H_{a}\right)=\mu\left(\underset{a \in S}{\cup} H_{a}\right) \leq \mu\left(\underset{a \in S}{\cup} G_{a}\right) \leq \sup \mu\left(\underset{\mathrm{a} \in S}{\cup} G_{a}\right),
$$

hence $\mu\left(\underset{\mathrm{a} \in A}{\cup} G_{\mathrm{a}}\right)=\sup \mu\left(\underset{\mathrm{a} \in J}{\cup} G_{\mathrm{a}}\right)$.

6. Theorem. Let $\Omega$ be a regular space whose $L$-weight is of measure zero. Then the following assertions are equivalent:

6.1. $\Omega$ is a Radon space of type ( $f)$.

6.2. Every subset of $\Omega$ which is universally Radon measurable of type $(f)$ is universally Borel measurable.

6.3. Every Spanish set of $\Omega$ is a universally Borel measurable set.

6.4. $\Omega$ is a Flock space.

Poof. $6.1 \Rightarrow 6.2$. Obvious.

$6.2 \Rightarrow 6.3$. It follows from the remark following definition 3 . $6.3 \Rightarrow 6.4$. From Corollary 2 .

$6.4 \Rightarrow 6.1$. Let $\Omega$ be a Flock space. Then we shall prove that $\Omega$ is $\mu$-compact if $\mu$ is a Borel measure on $\Omega$. Let $\left(G_{a}\right)_{a}$ be an open cover of $\Omega$; from Theorem 5 it follows that

$$
\mu(\Omega)=\mu\left(\cup_{a \in A} G_{a}\right)=\sup \underset{J}{\mu}\left(\cup_{a \in J} G_{a}\right) .
$$

Hence, for every $\varepsilon>0$, there exists a finite subset $J$ of $A$ such that $\mu\left(\Omega \backslash \cup G_{\mathrm{a}}\right)<\varepsilon$, so $\Omega$ is $\mu$-compact. In a similar way it is proved that each open $G$ of $\Omega^{\mathrm{a} \delta}$ is $\mu$-compact.

On the other hand, the class $\Sigma$ of the Borel sets $B$ such that

$$
\mu(B)=\sup \{\mu(F): B \supset F \in, \bar{J}\}
$$

and

$$
\mu\left(B^{c}\right)=\sup \left\{\mu(F): B^{c} \supset F \in . \ddot{f}\right\}
$$


is a $\sigma$-algebra which contains all the open subsets of $\Omega$. Indeed, if $\left(F_{a}\right)_{a \in A}$ is the family of the closed sets $F_{a} \subset G$, then $G=\underset{a \in A}{\cup} F_{a}^{\circ}$ since $\Omega$ is a regular space. So,

$$
\mu(G)=\sup \mu\left(\underset{a \in s}{\cup} F_{a}^{\circ}\right)=\sup \mu\left(\bigcup_{a \in J} F_{0}\right)
$$

and $G \in \Sigma$. Then every Borel set $B$ belongs to $\Sigma$ and $\Omega$ is a Radon space of type $(7)$.

Remark. If $\Omega$ is a Radon space of type ( $\mathscr{F}$ ) then the L-weight of $\Omega$ is of measure zero. If the L-weight is real-measurable, then there exists a well-ordered strictly increasing family $\left(G_{\mathrm{a}}\right)_{a \in A}$ of open sets such that card $(A)$ is realmesurable. Let $v$ be a Banach measure on $A$, select $x_{a} \in G_{a+1} \backslash G_{a}$ and set

$$
\mu(E)=U\left\{\alpha: x_{a} \in E\right\}(E \subset \Omega) .
$$

Then $\mu$ is a Borel measure but it is not a Radon measure of type (f): It were a Radon measure of type $(\mathscr{F})$, proceeding as in Proposition 1, would imply that $\mu\left(G_{\alpha}\right)=0$ for every $\alpha \in A$, and $v(A)=\mu\left(\cup_{\alpha \in A} G_{a}\right)=0$, and $v$ would not be a Banach measure.

The class of the Radon spaces of type (f), which contains the metrizable spaces whose weight is of measure zero and the strong Lindelof spaces, is very wide as it is proved in the following stability theorem.

\section{Theorem.}

7.1. If $\Omega$ is a Radon space of type (F), then every subset of $\Omega$ is a Radon space of type $(\Re)$.

7.2. If $\Omega$ is a regular space which is the union of a countable sequence $\left(E_{n}\right)$ of Radon subspaces of type (7), then $\Omega$ is a Radon space of type $(F)$.

7.3. If for every Borel measure $\mu$ and for every $\varepsilon>0$ there exists a Radon subspace of type $(\mathcal{F}) E_{\mathrm{a}} \subset \Omega$ such that $\mu^{*}\left(\Omega \backslash E_{2}\right)<\mathrm{E}$, where $\mu^{*}$ is the outer measure associated to $\mu$, then $\Omega$ is a Radon spaces of type $(\widehat{F})$.

Proof. 7.1 and 7.3 are immediate. To prove 7.2 it is sufficient to prove that $\Omega$ is $\mu$-compact, as in $6.4 \Rightarrow 6$.1. (See. Proposition $15[10]$ ).

8. Definition. A topological space (resp.uniform space) $\Omega$ is a said $\varepsilon$ metrizable (resp. uniform $\varepsilon$-metrizable) if, for every Borel measure $\mu$ on $\Omega$ and for every $\varepsilon>0$ there exists a metrizable set $E_{\mathrm{a}} \subset \Omega$ (resp. in the induced uniformity) such that $\mu^{*}\left(\Omega \backslash E_{2}\right)<\varepsilon$ where $\mu^{*}$ is the outer measure associated to $\mu$. 
By Theorems 6 and 7, every $\varepsilon$-metrizable space whose L-weight is of measure zero, is a Radon space of type $(j)$. Hence Theorem 7 could be completed by means of the following result: If $\left(E_{n}\right)$ is a sequence of $\varepsilon$-metrizable spaces, then the topological product $\prod_{1}^{\infty} E_{n}$ is a $\mathrm{\varepsilon}$-metrizable space.

Topological and uniform $\sigma$-metrizable spaces can be defined in a natural way. For them we can give an extension of a result of Marczewski and Sikorski:

9. Theorem. Let $\mu$ be a Borel measure on a uniform $\sigma$-metrizable space $\Omega$. Then the following assertions are equivalent:

9.1. There exists a decomposition $\Omega=F \cup N$, where $F$ is a separable closed set and $\mu(N)=0$.

9.2. $\mu$ is a Radon measure of type $\left(-\bar{s}_{s}\right)$, where $\dot{F}_{s}$ is the class of all the separable closed subsets of $\Omega$.

9.3. $\mu$ is a Radon measure of type $(j)$.

9.4. $\Omega$ is a $\mu$-compact space.

9.5. $\mu$ has a proper support, i.e, the union of all negligible open set is a negligible set.

Proof. cf. Theorem 12 [8].

The last theorem takes a more complete and stronger form if $E$ is a Banach space. In this case we can give new equivalent assertions.

In the following theorem, if $\Omega$ is a subset of a Banach space $E$, we will denote by $\left(\Omega\right.$, weak) the topological space $\left(\Omega, \sigma(E, E)_{\Omega \Omega}\right)$ and by $(\Omega$, norm) the topological space $\left(\Omega,\|.\|_{\Omega}\right)$.

10. Theorem. If $\Omega$ is a subset of the Banach space $E$, then the following assertions are equivalent:

10.1. For every Borel measure $\mu$ on $(\Omega$, weak) there exists a separable closed set $F$ such that $\mu(\Omega \backslash F)=0$.

10.2 For every Borel measure $\mu$ on $(\Omega$, weak), the completion $\bar{\mu}$ is a $R a$ don measure of type $(\%)$ on $\left(\Omega\right.$, norm), where $H_{0}$ is the class of the traces $K \cap \Omega$ of the compact sets $K$ of $E$.

10.3. Every Borel measure $\mu$ on $(\Omega$, weak $)$ is a Radon measure of type $\left(y_{1}\right)$, where $y_{1}$ is the class of the separable and metrizable (for the induced informity) closed subsets of $(\Omega$, weak).

10.4. $(\Omega$, weak $)$ is a Radon space of type $(\ni)$. 
10.5. ( $\Omega$, weak) is an $\varepsilon$-metrizable space whose L-weight is of measure zero.

10.6. ( $\Omega$, weak) is a Flock space with $L$-weight of measure zero.

10.7. Every Borel measure on $(\Omega$, weak) has a proper support $F$.

Proof. $10.1 \Rightarrow 10.2$. Let $\mu$ be a (finite) Borel measure on ( $\Omega$, weak). As $F$ is norm-separable, every Borel subset of $(F$, norm) is a Borel subset of $(F$, weak), hence the meassure $v$ defined on $E$ by $v(B)=\mu(B \cap F)$ is a Radon measure of type (f), so, it is a Radon measure because $E$ is universally measurable. Then, $\bar{\mu}$ is a Radon measure of type $\left(., 7_{0}\right)$ since $\mu(\Omega \backslash F)=0$.

$10.2 \Rightarrow 10.3$. It is sufficient to prove that each $H \in \varkappa_{0}$ is metrizable, for the induced uniformity, and separable in $(\Omega$, weak).

$10.3 \Rightarrow 10.4$. Obvious.

$10.4 \Rightarrow 10.7$. Clear.

$10.3 \Rightarrow 10.5$. From the remark following Theorem 6 and from 10.4, the L-weight of $\Omega$ is of measure zero. Then 10.5 follows directly from 10.3 .

$10.5 \Rightarrow 10.6$. cf. Theorems 6 and 7 .

$10.6 \Rightarrow 10.4$. cf Theorem 6 .

$10.7 \Rightarrow 10.1$. Since $\Phi=\left\{x_{, \mu}^{*} x^{*} \in E^{*},\left\|x^{*}\right\| \leq 1\right\}$ is a convex set of measurable functions which is compact in the topology $\tau_{p}$ of pointwise convergence, and Hausdorff in the topology $\tau_{m}$ of convergence in measure, from A. Bellow's Theorem 12.3.3 [36], it follows that $\Phi$ is metrizable in $\tau_{p}=\tau_{m}$. Hence, $(F$, norm) is separable.

Remark. The last theorem can be completed by the use of the fact that a Radon space of type $(\bar{f})$ is a Radon space if and only if it is universally measurable.

\section{COMPLETELY ADDITIVE OF MEASURABLE SETS}

We are going to study the case when the union of a family $\left(E_{a}\right)_{a \in A}$ of measurable sets is measurable.

11. Definition. $A$ family $\left(E_{\mathrm{a}}\right)_{a \in A}$ of subsets of $\Omega$ is said to be relatively discrete if every point of $\cup E_{\mathrm{a}}$ has a neighborhood which meets exactly one member of the family. The family will be said discrete in $\Omega$ if each point of $\Omega$ has a neighborhood which meets at most one member of the family. 
Let $M$ be a set of complete Radon measures of type ( $A$ ) (or $\tau$-additive) on $\Omega$. A family $\left(E_{\alpha}\right)_{\alpha \in A}$ of subsets of $\Omega$ is said to have an a.e. $\sigma$-discrete decomposition (a.e. $\sigma$-d.d.)(resp. $\sigma$-relatively discrete, a.e. $\sigma$-r.d.d.) with respect to $M$ if, for every measure $\mu \in M$, each

$$
E_{\mathrm{a}}=\bigcup_{n=1}^{\infty} E_{\mathrm{an}} \cup Z_{\mathrm{a}},
$$

where every $\left(E_{a n}\right)_{a \in A}$ is discrete (resp. relatively discrete) and $\mu\left(\cup Z_{a}\right)=0$.

From now on all measures will be finite and complete Radon measures of type (f).

Preceding concepts coincide as we prove in the following

12. Proposition. The family $\left(E_{\alpha}\right)_{\mathrm{a} \in A}$ of subsets of $\Omega$ has a a.e. $\sigma-r . d . d$. with respect to $\mu$ if and only if there exists a countable subset $A_{o} \subset A$ such that $\mu\left(\bigcup_{\mathrm{a} \in A_{0}} E_{\mathrm{a}}\right)=0$.

Proof. Let $E_{\mathrm{a}}=\cup E_{a n} \cup Z_{\mathrm{a}}$ where every $\left(E_{a \mathrm{an}}\right)_{\mathrm{a} f \mathrm{~A}}$ is relatively discrete and $\mu\left(\cup Z_{\mathrm{a}}\right)=0$. Then, for every $\alpha \in A$ and $n \in \mathbb{N}$, there exists an open set $G_{\mathrm{an}}$ such that $E_{\mathrm{an}} \subset G_{\mathrm{an}}$ and $E_{\mathrm{a}^{\prime n} \cap} \cap G_{\mathrm{an}}=\emptyset$ when $\alpha^{\prime} \neq \alpha$. Hence

$$
\sum_{\alpha \in A} \mu^{*}\left(E_{a n}\right) \leq \mu(\Omega)<\infty
$$

for each $n \in \mathbb{N}$, so there exists a countable set $A_{n} \subset A$ such that $\mu\left(E_{\text {an }}\right)=0$ for each $a \in A_{n}^{c}$. As every open is $\mu$-compact and

$$
\left(\underset{a \in A_{n}^{e}}{\cup_{a n}}\right) \cap G_{a n}=E_{a n}
$$

when $\alpha \in A_{\mathrm{n}}^{\mathrm{c}}$ it results that

$$
\mu\left(\underset{a \in A_{n}^{c}}{\cup} E_{a n}\right)=\mu\left(\left(\cup_{a \in A_{n}^{c}} E_{a n}\right) \cap\left(\underset{a \in A_{n}^{c}}{\cup} G_{a n}\right)\right)=0
$$

Let $A_{\mathrm{o}}=\cup_{n \in \mathrm{N}} A_{n}$, then $A_{\mathrm{o}}$ is countable and

$$
\mu\left(\underset{a \in S_{o}^{c}}{\cup} E_{\alpha}\right) \leq \sum_{n \in \mathbb{N}} \mu\left(\underset{a \in A_{n}^{c}}{\cup} E_{a n}\right)+\mu\left(\underset{\alpha \in A}{\cup} Z_{\alpha}\right)=0 .
$$

The converse is immediate.

13. Definition. $A$ family $\left(E_{\mathrm{a}}\right)_{\text {aed }}$ of subsets of $\Omega$ is said $\tau$-additive with respect to $\mu$ if, for every $A^{\prime} \subset A$, there exists a countable subset $A_{o} \subset A^{\prime}$ such that

$$
\mu\left(\underset{a \in A}{\cup} E_{\mathrm{a}} \backslash \underset{\mathrm{a} \in A_{o}}{\cup} E_{\mathrm{a}}\right)=0
$$


From Proposition 12 it follows that every family a.e.o-d.d. is $\tau$-additive. It is clear the family $G$ of all the open sets of $\Omega$ is $\tau$-additive with respect to each Radon measure of type (- $)$, (or $\tau$-additive). If $E$ is a Radon space of type $(F), \mathscr{\gamma}$ the topology on $E$, and $f \Omega \rightarrow E$ a Borel $\mu$-measurable function, then $\left\{f^{-l}(V): V \in \mathcal{Y}\right\}$ is a $\tau$-additive family with respect to $\mu$.

14. Proposition. Let $\left(E_{\mathrm{a}}\right)_{\mathrm{a} \in A}$ be a locally countable family of subsets of $\Omega$ and $\mu$ a measure on $\Omega$ then $\left(E_{\alpha}\right)_{a \in A}$ is a.e. $\sigma-d . d$. with respect to $\mu$. Hence, every locally countable family is a.e. $\sigma-d . d$.

Proof. In fact, for every $x \in \cup E_{\mathrm{a}}$, there exists a neighborhood $V(x)$ and a countable subset $A_{x} \subset A$ such that $V(x) \cap E_{\mathrm{a}}=\varnothing$ for every $\alpha \notin A_{x}$. As $\mu$ is a Radon measure of type $\left(x_{n}\right)$ there exists a sequence $\left(V\left(x_{n}\right)\right.$ sut

$$
\mu\left(\cup_{\mathbf{a} \in A} E_{\mathrm{a}} \backslash \underset{n \in \mathbb{N}}{\cup} V\left(x_{n}\right)\right)=0 .
$$

Let $A_{o}=\cup_{n \in \mathbb{N}} A_{x_{n}}$, then $A_{o}$ is countable and

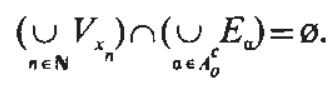

so,

$$
\left.\mu\left(\underset{\mathrm{a} \in A_{0}^{c}}{\cup} E_{\mathrm{a}}\right) \leq \mu\left(\underset{\mathrm{a} \in \mathrm{A}}{\cup} E_{\mathrm{o}}\right) \underset{n \in \mathbb{N}}{\cup} V_{x_{n}}\right)=0
$$

and we conclude that $\left(E_{a}\right)_{a \in A}$ is a.e. $\sigma$-d.d. with respect of $\mu$.

15. Theorem. If $\left(E_{\alpha}\right)_{a \in A}$ is an a.e.o-d.d. family (with respect to $\mu$ ) of $\mu$ measurable sets, then $\cup_{a \in A^{\prime}} E_{\mathrm{a}}$ is a $\mu$-measurable set for every $A^{\prime} \subset A$.

Proof. In fact, $\left(E_{a}\right)_{a \in A}$ is $\tau$-additive by Proposition 12 .

16. Theorem. Let $\mu$ be a Radon measure of type $\left(-x_{m}\right)$ and $\left(E_{0}\right)_{0 \in A}$ a family of subsets of $\Omega$ such that, for every $A^{\prime} \subset A, \cup E_{a}$ is $\mu$-measurable. Then, if

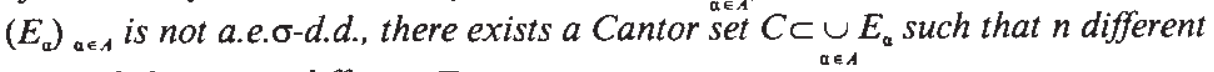
points belong to $n$ different $E_{\mathrm{a}}$.

The proof is based on Theorem 2 [16] due Hansell and can be found in Theorem 26 [10]. As a consequence

17. Corollary. If $\mu$ a Radon measure of type $\left(\mathscr{H}_{\mathrm{m}}\right.$ ) on $\Omega$, then one (and only one) of the following assertion is true:

(i) $\Omega$ is a.e. $\sigma-d . d$., i.e., there exists a $\sigma$-discrete subset $E_{o}$ such that $\mu\left(\Omega \backslash E_{o}\right)=0$. 
(ii) $\Omega$ contains a subset homeomorphic to the Cantor subset and also contains another subset not $\mu$-measurable.

This result is analogous to a well-know result of A.H. Stone and A.G. El'kin [34] [11], and it can be proved as Hansell does in [16].

The complete additivity of a family of measurable sets can be studies by means of the following:

18. Theorem. Let $\mu$ be a perfect measure on $\Omega$ and $\left(E_{\alpha}\right)_{a \in A}$ a disjoint family of subsets of $\Omega$ such that $\mu\left(E_{\alpha}\right)=0$ for every $\alpha \in A$. Then one and only one of the following cases is verified:

(i) For every $A^{\prime} \subset A$, the union $\cup E_{\mathrm{a}}$ is $\mu$-measurable and there exists a countable partition $\left(A_{n}\right)$ of $A$ such that

$$
\left\{\mu\left(\cup E_{a}\right): A^{\prime} \subset A_{n}\right\}=\left\{0, \mu\left(\underset{a \in A_{n}^{\prime}}{\cup} E_{a}\right)\right\}
$$

for every $n$.

(ii) There exists $A^{\prime} \subset A$ such that $\underset{\mathrm{a} \in A^{\prime}}{\cup} E_{\mathrm{a}}$ is not $\mu$-measurable.

Proof. Similar to Theorem 2.5 [21] where it is supposed that the cardinal of $A$ is not measurable.

Remark If $\mu \neq 0$ is a Radon measurable of type ( $j$, then 18(i) is not verified. In fact, let us suppose 18(i) and

$$
\left\{\mu\left(\underset{\mathrm{a} \in \mathrm{A}^{\prime}}{\cup} E_{\mathrm{a}}\right): A^{\prime} \subset \mathrm{A}\right\}=\{0,1\} .
$$

Then, if we take $x_{\mathrm{a}} \in E_{\mathrm{a}}$ (when $E_{\mathrm{a}} \neq \emptyset$ ) and we define

$$
v(E)=\mu\left(\cup\left\{E_{\mathrm{a}}: x_{\mathrm{a}} \in E\right\}\right),
$$

$\mu$ is a continuous Ulam measure. As $\mu$ is t-additive, every $x \in \Omega$ is a neighborhood $V(x)$ such that $\mathrm{v}(V(x))=0$, and it results that $\mathrm{v}(\Omega)=0$ and this is a contradiction with

$$
\mathrm{v}(\Omega)=\mu\left(\underset{a \in A}{\cup} E_{\mathrm{a}}\right)=1 .
$$

19 Theorem. Let $\mu$ be a perfect Radon measure of type $(-\bar{x})$ on $\Omega$. If $\left(E_{\mathrm{a}}\right)_{\mathrm{a}, \mathrm{A}}$ is a disjoint family of $\mu$-measurable subsets of $\Omega$, then one and only one of the following cases is true:

(i) $\left(E_{\alpha}\right)_{a \in d}$ is a.e. $\sigma-d . d$. with respect to $\mu$. 
(ii) There exists $A^{\prime} \subset A$ such that $\cup_{\mathrm{a} \in A^{\prime}} E_{\mathrm{a}}$ is not $\mu$-measurable.

Proof. If follows from theorem 18 and the above remark applied to the family

$$
\left\{E_{\mathrm{a}:}: \mu\left(E_{\mathrm{a}}\right)=0, \alpha \in A\right\} .
$$

For Radon measures, Fremlim has proven the following theorem:

20 Theorem (Fremlim, [13]). Let $\mu$ be a Radon measure on $\Omega$ and let $\left(E_{\mathrm{a}}\right)_{a \in A}$ be a point-finite family of $\mu$-measurable sets. Then one and only one of the following assertion in true:

(i) $\left(E_{a}\right)_{a \in A}$ is $\tau$-additive with respect to $\mu$.

(ii) There exists $A^{\prime} \subset A$ such that $\underset{a \in A^{\prime}}{\cup} E_{a}$ is not $\mu$-measurable.

With Martin's Axiom, Fremlim proved in [13] that the condition of pointfiniteness could be changed by point-countability.

21. Theorem. Let $\mu$ be a Radon measure of type ( $\not \vec{\jmath})$ on $\Omega$ and $\left(E_{\alpha}\right)_{\text {aed }}$ a locally countable family of $\mu$-measurable subsets. Then $\left(E_{\mathrm{a}}\right)_{\mathrm{a} \in \mathrm{A}}$ is a.e. $\sigma-d . d$. with respect to $\mu$ and $\cup E_{a}$ is $\mu$-measurable for each $A^{\prime} \subset A$.

Proof. cf. Proposition 14.

Remark: The results of the present paper can be applied to study the Borel measurable functions $f \Omega \rightarrow E$ since, if $r$ is the topology of $E$, the union of every subfamily of $\left\{f^{-1}(V): V \in \mathscr{T}\right\}$ is measurable. (See, [10]).

\section{References}

[1] ALEXANDrofF, A. D. Additive set functions in abstract spaces. Mat. Sb. 8 (1940) 307-348; $9(1941), 169-238$.

[2] Bourbaki, N.: Integration, Chap 1-9. Paris, Hermann, 1960-1969.

[3] CARATHEODORY, C.: Vorlesungen über reelle Funktionen. Leipzig-Berlin, Teubner, $(1918,1927)$.

[4] DANIEL, P.J.: A general form of integral. Ann. of Math. 19(1917), 279-294.

[5] DANIEL, P.J.: Integral in an infinite number of dimensions. Ann. of Math.20 (1918-1919), 281-288.

[6] DANIEL, P.J.: Functions of a limited variation in an infinite number of dimensions. Ann. of Math. 21 (1919-1920), 30-38. 
[7] De Maria, J. and Rodriguez-Salinas, B.: On Measures on Metric Spaces. (1987). Preprint.

[8] De Maria, J. and Rodriguez-Salinas, B.: On measures on $\sigma$-metrizable spaces. (1988). Preprint.

[9] De MARIA, J. and RODRIGUEZ-Salinas, B.: On measures on Banach spaces with the weak topology. (1988). Preprint.

[10] DE MARIA, J. and RODRIGUEZ-SALINAS, B.: On measurable sets of a $\tau$-additive measure. (1988). Preprint.

[11] EL'KIN, A. G.: A-sets in complete metric spaces. Dokl. Akad. Nauk. SSSR. 175, (1967), 517-520. Soviet. Math. Dokl. 8 (1967), 874-877.

[12] Fremlin, D. H.: Topological Riesz Spaces and Measure Theory. Cambridge University Press. London, 1974.

[13] FREMLIN, D. H.: On measurable selections. Preprint.

[14] Gardner, R. J.: The regularity of Borel measures. Proc. Measure Theory Conf. Oberwolfach, (1981) Lect. Notes in Math. 945 (1981), $42-100$.

[15] Halmos, P. R.: Measure Theory. Van Nostrand, New York, 1950.

[16] HANSELL, R. W.: Borel measurable mapping for non-separable metric spaces. Trans. Amer. Math. Soc. 161 (1971), 145-169.

[17] HANSELl, R. W.: On Borel Mapping and Baire Functions. Trans. Amer. Math. Soc. 194 (1974), 195-211.

[18] JESSEN, B.: The theory of integration in a space of a infinite number of dimensions. Acta Math. 63 (1934), 249-323.

[19] Jimenez GuerRa, P. and Rodriguez-SAlinas, B.:Espacios de Radon de tipo (y/). Rev. R. Acad. Cienc. Exactas Fis. Natur. Madrid, 69 (1975), $761-774$.

[20] Jimenez Guerra, P. and Rodriguez-Salinas, B: Medidas de Radon de tipo (yt) en espacios topologicos arbitrarios. Mem. Acad. Cien. Exactas. Fis. Nat. Madrid, (1979).

[21] Koumoulis, G.: On perfect measures. Trans. Amer. Math. Soc. 264. (1981) 521-537.

[22] LE CAM, L.: Convergence in distribution of stochastic processes. Univ. Cal. Pub. Statistic 2,11 (1957), 207-236.

[23] MARCZEWSKI, E and SIKORSKI, R: Measures on nonseparable metric spaces. Colloq. Math. 1 (1948), 133-139.

[24] MINLos, R. A.: Generalized randon processes and their extension to a measture. Trudy Mosk. Mat. Obsc. 8 (1959), 497-518. English trad. Selected translation in math. satistic and probability, 3 (1960), 291-313.

[25] MONTGOMERY, D: Non-separable Metric Spaces. Fund. Math. 25 (1935), 527-533.

[26] PROKHOROV, YU. V: Convergence of random processes and limit theorems in probability theory. Theor. Prob. Appl. 1 (1956), 157-214.

[27] RoDRIGUEZ-SAlinas, B.: Construccion de las medidas de Borel sobre los espacios regulares. Actas de la V Reunion Anual de Matemáticos Españoles. Madrid (1964), 34-41. 
[28] Rodriguez-Salinas, B.: Teoria de la medida sobre espacios topologicos no localmente compactos. Rev. Mat Hispano Americana (4)32(1973), 257-274.

[29] ROdRIGUeZ-Salinas,B.: $\mu$-espacios de Suslin y Lusin. Propiedad del "lifting" fuerte. Rev. Real. Acad. Cienc. Exactas. Fis. Natur. Madrid, 72 (1978), 541-557.

[30] RodrigueZ-SaLinas, B.: Perfect measures spaces and coperfect spaces. (1987). Preprint.

[31] SCHWARTZ, L.: Measures de Radon sur des espaces non localement compact. Theory of distributions. (Proc. Intern. Summer Inst., Lisbon 1964) 3-22. Inst. Gulbenkian $\mathrm{Ci}$, Lisbon, 1964.

[32] SCHWARTZ, L: Radon Measures on arbitrary Topological Spaces and Cylindrical Measures. Oxford Univ. Press. 1973.

[33] STEInHAuS, V.M.: Les probabilités dénombrables et leur rapport à la théory de la mesure. Fund. Math., 4(1923), 286-310.

[34] STONE, A.H.: On б-discreteness and Borel isomorphism. Amer. J. Math., 85 (1963), 656-666.

[35] STONE, A.H. Some problems of Measurability. Lect. Notes in Math. 375 (1974), 242-248.

[36] Talagrand, M.: Pettis Integral and Measure Theory. Mem Amer. Math. Soc. 307 (1984).

[37] UlaM, S.: Zur Mass theorie inder allgemeinen Mengenlehre. Fund. Math., 16 (1930), 140-150.

[38] VARadarajan, V. S.: Measures on topological Spaces. Amer. Math. Soc. Transl. (2) 48 (1965), 161-228.

[39] WheEler, R.A.: Survey of Baire Measures and Strict Topologies. Memory of Depart. of Math. Sciences. Northern Illinois University.

Departamento de

Matemáticas Fundamentales

U.N.E.D.

Ciudad Universitaria

MADRID-28040-SPAIN
Departamento de Análisis Matemático Facultad de Matemáticas Universidad Complutense MADRID MADRID-28040-SPAIN 